\title{
SYNTHESIS OF 1-ARYL-6,6-DIMETHYL-2-PHENYL-6,7-DIHYDRO-1H-INDOL-4(5H)-ONES BY TWO STEPS, IN A THREE-COMPONENT REACTION
}

\author{
JABBAR KHALAFY*, FAHIMEH BADPARVAR, AHMAD POURSATTAR MARJANI \\ Department of Chemistry, Faculty of Science, Urmia University, Urmia 57154, Iran
}

\begin{abstract}
An efficient method for the synthesis of 1-aryl-6,6-dimethyl-2-phenyl-6,7-dihydro-1H-indol-4(5H)-ones is achieved in two steps, using a three-component reaction of phenacyl bromide, dimedone and aniline derivatives in water-ethanol (1:1) under reflux conditions.
\end{abstract}

Keywords: Phenacyl bromide, Dimedone, Anilines, $1 H$-Indol-4(5H)-ones, Three-component reaction.

\section{INTRODUCTION}

In recent years, nitrogen-containing heterocyclic compounds have been the focus of both chemists and biochemists due to their wide range of biological and pharmaceutical properties. ${ }^{1,2}$

Among the various classes of nitrogen-containing heterocycles, indoles have many biological and pharmacological activities, such as GABA receptor ligands, ${ }^{3}$ antipsychotic, ${ }^{4}$ anticancer, ${ }^{5}$ antioxidant, ${ }^{6}$ antiproliferative agents, ${ }^{7}$ antirheumatoidal and anti-HIV, ${ }^{8,9}$ and as soluble guanylatecyclase inhibitors. ${ }^{10,1}$ Therefore, the synthesis and selective functionalization of indoles ${ }^{12-14}$ and using indoles as starting materials for the synthesis of a series of alkaloids, pharmaceuticals and perfumes ${ }^{15}$ have been the focus of researchers. A number of these are costly and harmful to the environment. By contrast, few synthetic methods based on green chemistry have been reported. ${ }^{16-18}$

None the less, the synthesis of indoles in aqueous or less toxic solvents by reducing the stages of the synthetic routes has been reported. ${ }^{19}$ Multi-component reactions (MCRs) are powerful methods for the synthesis of organic compounds ${ }^{20}$ and are defined as convergent chemical processes where three or more reagents are combined in such a way that the final product retains significant portions of all starting materials. They lead to the connection of three or more starting materials in a single synthetic operation with high atom economy and bond-forming efficiency, thereby increasing molecular diversity and complexity in a fast and often operational simplicity. ${ }^{21-22}$

A facile synthesis of substituted 6,7-dihydro- $1 \mathrm{H}$-indol-4(5H)-ones by reaction of cyclohex-2-enone and 2-oxo-2-(substituted phenyl)acetaldehyde has recently been reported. ${ }^{23}$ In addition, Kaladevi and co-workers reported the reaction of 1-aryl-2-arylaminoethanones with substituted cyclohexane-1,3diones in acetic acid through an intramolecular cyclization to form 1,3-diaryl-6,7-dihydro- $1 H$-indol-4(5H)-ones. ${ }^{24}$ In continuation of our interest in multicomponent synthesis of nitrogen-containing heterocyclic compound, ${ }^{25}$ herein we report the synthesis of 1-aryl-6,6-dimethyl-2-phenyl-6,7-dihydro- $1 H$-indol$4(5 H)$-ones by two steps, involving a three-component reaction of phenacyl bromide, dimedone and various anilines in the presence of $\mathrm{K}_{2} \mathrm{CO}_{3}$ in waterethanol $(1: 1)$ under reflux conditions.

\section{RESULTS AND DISCUSSION}

5,5-Dimethylcyclohexane-1,3-dione (2) was alkylated with phenacyl bromide (1), prepared from acetophenone via bromination with bromine in acetic acid at room temperature, ${ }^{26}$ in the presence of $\mathrm{K}_{2} \mathrm{CO}_{3}$, to give the triketone ${ }^{27}$ (3). (Scheme 1)

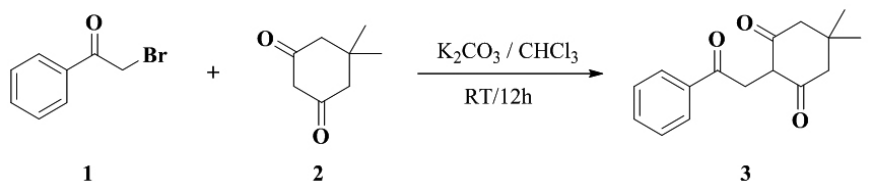

Scheme 1. Preparation of triketone (3)

The desired indoles were synthesized by adding anilines (4a-g) to triketon (3) in water-ethanol (1:1) and refluxing the reaction mixture overnight (Scheme 2 ). The products $(\mathbf{5 a}-\mathbf{g})$ were obtained in good yields after recrystallization from methanol-acetone $(1: 1)$. The list of products $(\mathbf{5 a - g})$ along with their yields and melting points are given in Table 1.

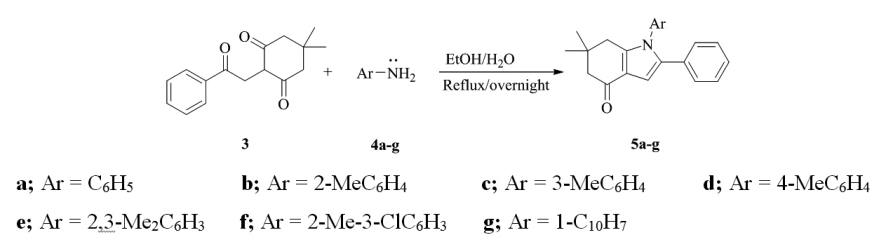

Scheme 2. Synthesis of $1 H$-indol-4(5H)-one derivatives

The improved procedure reported in this article is a simple and environmentally benign in comparison with other method mentioned in the literature.

The spectral data for all products is consistent with the assigned structures. In particular, in the ${ }^{1} \mathrm{H}-\mathrm{NMR}$ spectra of the products, the indole $3-\mathrm{H}$ is characterized by a singlet at $\delta=6.79-6.93 \mathrm{ppm}$. The $\mathrm{CH}_{2}$ next to the carbonyl group resonated as singlets at $\delta=1.86-2.42 \mathrm{ppm}$ while the $\mathrm{CH}_{2}$ next to the pyrrole ring appear as singlets at $\delta=2.12-2.53 \mathrm{ppm}$. Each of the two methyl groups on the aliphatic ring appear as singlets at $\delta=0.85-1.27 \mathrm{ppm}$.

The ${ }^{13} \mathrm{C}$-NMR spectra are characterized by the indole $3 \mathrm{C}$ reonances at $\delta$ 105.65 (5a), 110.34 (5b), 110.07 (5c), 104.87 (5e), 110.00 (5f), 105.21 (5g) (ppm). The $\mathrm{CH}_{2}$ next to the carbonyl group resonates at $\delta 52.13(5 \mathrm{a}), 46.13$ $(5 \mathrm{~b}), 46.29(5 \mathrm{c}), 52.20(5 \mathrm{e}), 46.37(5 \mathrm{f}), 52.21(5 \mathrm{~g})(\mathrm{ppm})$. The $\mathrm{C}-7 \mathrm{CH}_{2}$ appeared at $\delta 37.08(5 \mathrm{a}), 31.74(5 \mathrm{~b}), 31.74(5 \mathrm{c}), 36.59(5 \mathrm{e}), 31.71(5 \mathrm{f}), 36.38^{2}(5 \mathrm{~g})$ (ppm). The $\mathrm{C}=\mathrm{O}$ absorption in the FT-IR spectra were observed at 1610-1660 $\mathrm{cm}^{-1}$.

\section{EXPERIMENTAL}

Infrared spectra were recorded on a Thermo Nicolet Nexus 670 FT-IR instrument using $\mathrm{KBr}$ discs. ${ }^{1} \mathrm{H}$ and ${ }^{13} \mathrm{C}$ NMR spectra were recorded on a Bruker Avance AQS $300 \mathrm{MHz}$ spectrometer at 300 and $75.5 \mathrm{MHz}$, respectively. Chemical shifts were measured in $\mathrm{CDCl}_{3}$ relative to TMS as the internal standard. Elemental analyses were performed by using a Leco Analyzer 932. Melting points were measured on a Philip Harris C4954718 apparatus and are uncorrected.

\section{Preparation of triketone (3):}

Phenacyl bromide ${ }^{26}$ (1 mmol), 5,5-dimethylcyclohexane-1,3-dione (1 $\mathrm{mmol})$ and anhydrous $\mathrm{K}_{2} \mathrm{CO}_{3}(0.13 \mathrm{mmol})$ were stirred together in dry $\mathrm{CHCl}_{3}(8$ $\mathrm{mL}$ ) for $12 \mathrm{~h}$ at $25^{\circ} \mathrm{C}$. The mixture was filtered and the residual potassium salt of the product was dissolved in water $(60 \mathrm{~mL})$, and the solution was acidified with $\mathrm{HCl}$. The crude product $(82 \%)^{28}$ was collected, washed with water, and recrystallized from EtOH, m.p. $88^{\circ} \mathrm{C}$.

General Procedure for the synthesis of 1-aryl-6,6-dimethyl-2-phenyl6,7-dihydro- $1 H$-indol-4(5H)-ones (5a-g):

The aniline $(2 \mathrm{mmol})$ was added to a solution of triketone $(3)(1 \mathrm{mmol})$ in water-ethanol $(1: 1)(10 \mathrm{~mL})$ and heated under reflux conditions overnight. The reaction mixture was cooled to room temperature and the product was then collected as a light pink precipitate which was washed with cold aqueous ethanol and purified by recrystallization from methanol-acetone (1:1) to give the desired products $(\mathbf{5 a - g})$ in $73-94 \%$ yield. 
Table 1. The yields and melting points of products (5a-g).

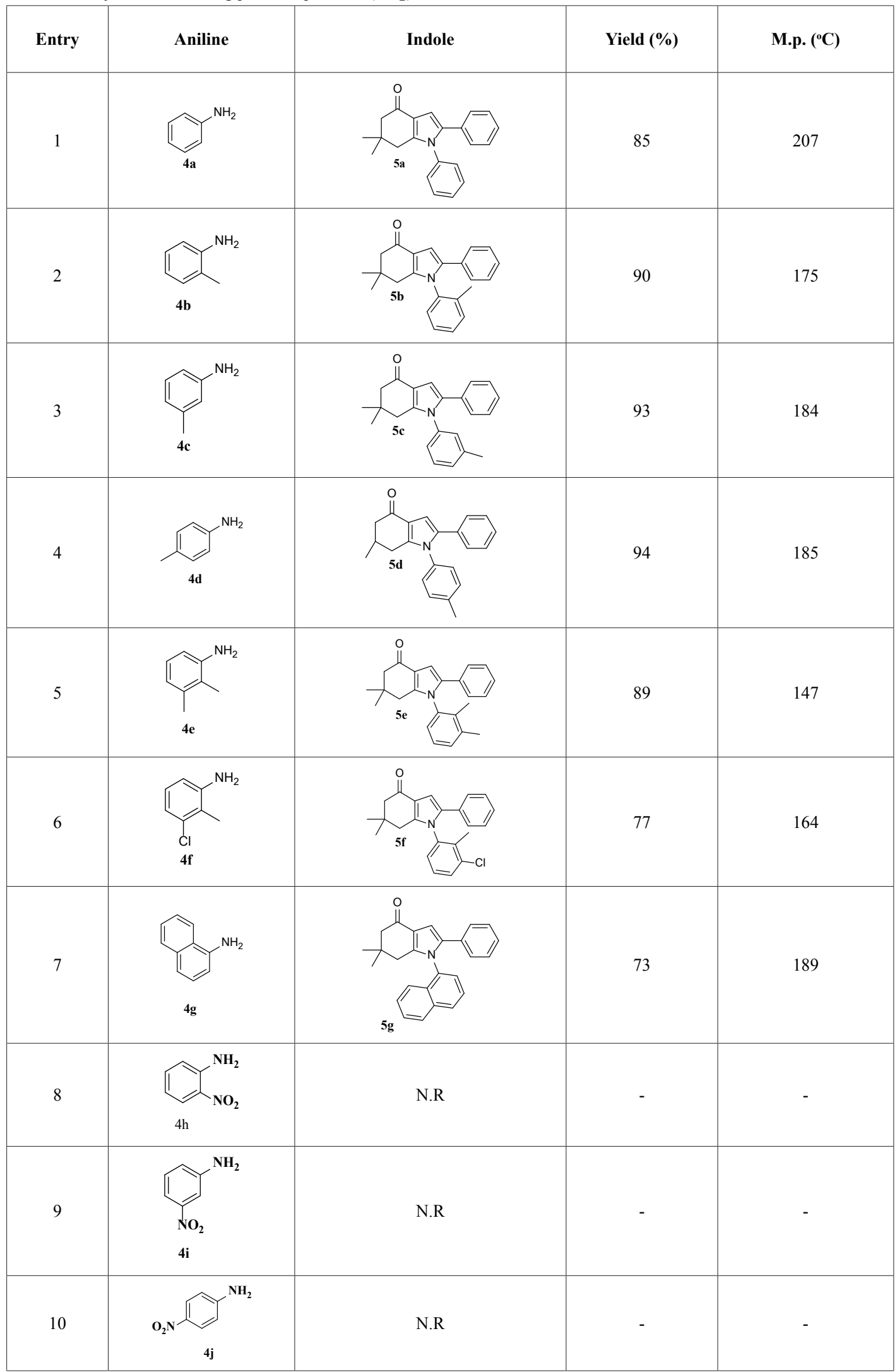


6,6-Dimethyl-1,2-diphenyl-6,7-dihydro-1 $H$-indol-4(5H)-one (5a):

White solid, yield $85 \%$, m.p. $207{ }^{\circ} \mathrm{C}$ [Lit. ${ }^{29} 20{ }^{\circ} \mathrm{C}$ ]. ${ }^{1} \mathrm{H}-\mathrm{NMR}(300 \mathrm{MHz}$, $\left.\mathrm{CDCl}_{3}\right) \delta(\mathrm{ppm}): 1.06(\mathrm{~s}, 3 \mathrm{H}, \mathrm{Me}), 1.10(\mathrm{~s}, 3 \mathrm{H}, \mathrm{Me}), 2.42\left(\mathrm{~s}, 2 \mathrm{H}, \mathrm{CH}_{2}\right), 2.53$ $\left(\mathrm{s}, 2 \mathrm{H}_{1} \mathrm{CH}_{2}\right), 6.79(\mathrm{~s}, 1 \mathrm{H}, \mathrm{Ar}), 7.04-7.10(\mathrm{~m}, 2 \mathrm{H}, \mathrm{Ar}), 7.11-7.21(\mathrm{~m}, 5 \mathrm{H}, \mathrm{Ar})$, 7.37-7.44 (m, 3H, Ar). ${ }^{13} \mathrm{C}-\mathrm{NMR}\left(75.5 \mathrm{MHz}, \mathrm{CDCl}_{3}\right) \delta(\mathrm{ppm}): 28.64,35.56$, $37.08,52.13,105.65,119.94,126.87,127.77,128.14,128.18,128.26,128.61$, $129.34,131.93,136.33,137.70,144.71,193.99$. FT-IR $v_{\max } 3058,2956,2875$ $1620,1551,1493,1394,1327,1151,1111,771,698 \mathrm{~cm}^{-1}$. (5b):

6,6-Dimethyl-2-phenyl-1-(o-tolyl)-6,7-dihydro-1 $H$-indol-4(5H)-one

Light pink solid, yield $90 \%$, m.p. $175^{\circ} \mathrm{C}\left[\right.$ Lit. $\left.^{30} 174-176{ }^{\circ} \mathrm{C}\right] .{ }^{1} \mathrm{H}-\mathrm{NMR}$ $\left(300 \mathrm{MHz}, \mathrm{CDCl}_{3}\right) \delta(\mathrm{ppm}): 0.89(\mathrm{~s}, 3 \mathrm{H}, \mathrm{Me}), 1.05(\mathrm{~s}, 3 \mathrm{H}, \mathrm{Me}), 1.86(\mathrm{~s}, 2 \mathrm{H}$, $\left.\mathrm{CH}_{2}\right), 2.17\left(\mathrm{~s}, 2 \mathrm{H}, \mathrm{CH}_{2}\right), 2.23(\mathrm{~s}, 3 \mathrm{H}, \mathrm{Me}), 6.90-7.20(\mathrm{~m}, 7 \mathrm{H}, \mathrm{Ar}), 7.20-7.33(\mathrm{~m}$, $3 \mathrm{H}, \mathrm{Ar}) .{ }^{13} \mathrm{C}-\mathrm{NMR}\left(75.5 \mathrm{MHz}, \mathrm{CDCl}_{3}\right) \delta(\mathrm{ppm}): 17.61,27.59,28.72,31.74$, $46.13,108.32,110.34,112.27,125.72,127.01,127.56,128.11,129.72,130.09$, $130.38,131.76,134.93,136.55,137.72,139.56,185.39$. FT-IR $v_{\max } 3056,2954$, $2872,1626,1550,1490,1394,1328,1150,1109,768,700 \mathrm{~cm}^{-1}$. (5c):

6,6-Dimethyl-2-phenyl-1-(m-tolyl)-6,7-dihydro-1 $H$-indol-4(5H)-one

Light pink solid, yield $93 \%$, m.p. $184^{\circ} \mathrm{C}\left[\right.$ Lit. $\left.^{30} 184-185^{\circ} \mathrm{C}\right] .{ }^{1} \mathrm{H}-\mathrm{NMR}(300$ $\left.\mathrm{MHz}, \mathrm{CDCl}_{3}\right) \delta$ (ppm): 0.89 (s, 3H, Me), 1.05 (s, 3H, Me), 2.12 (s, 2H, $\mathrm{CH}_{2}$ ) $2.26(\mathrm{~s}, 5 \mathrm{H}, \mathrm{CH}+\mathrm{Me}), 6.79(\mathrm{~d}, J=6.9 \mathrm{~Hz}, 1 \mathrm{H}, \mathrm{Ar}), 6.80(\mathrm{~s}, 1 \mathrm{H}, \mathrm{Ar}), 6.92(\mathrm{~d}$ $J=7.2 \mathrm{~Hz}, 1 \mathrm{H}, \mathrm{Ar}), 6.95-7.03$ (m, 4H, Ar), 7.05-7.16 (m, 3H, Ar). ${ }^{13} \mathrm{C}-\mathrm{NMR}$ $\left(75.5 \mathrm{MHz}, \mathrm{CDCl}_{3}\right) \delta(\mathrm{ppm}): 21.08,27.57,28.69,30.08,31.74,46.29,110.07$, $112.63,125.75,126.90,127.56,127.64,129.37,127.99,130.01,130.44$, $132.02,134.48,138.14,138.25,186.44$. FT-IR $v_{\max } 3056,2953,1610,1548$, $1487,1398,1340,1242,1031,763,696 \mathrm{~cm}^{-1}$ (5d):

6,6-Dimethyl-2-phenyl-1-(p-tolyl)-6,7-dihydro- $1 H$-indol-4(5H)-one

White solid, yield $94 \%$, m.p. $185^{\circ} \mathrm{C}$ [Lit. $\left.{ }^{30} 184-187{ }^{\circ} \mathrm{C}\right] .{ }^{1} \mathrm{H}-\mathrm{NMR}(300$ $\mathrm{MHz}, \mathrm{CDCl}_{3}$ ) $\delta$ (ppm): 1.11 (s, 3H, Me), 1.27 (s, 3H, Me), 2.41 (s, 3H, Me), $2.43\left(\mathrm{~s}, 2 \mathrm{H}, \mathrm{CH}_{2}\right), 2.52\left(\mathrm{~s}, 2 \mathrm{H}, \mathrm{CH}_{2}\right), 6.79(\mathrm{~s}, 1 \mathrm{H}, \mathrm{Ar}), 7.02-7.05(\mathrm{~m}, 2 \mathrm{H}, \mathrm{Ar})$, 7.08-7.10 (m, 2H, Ar), 7.17-7.23 (m, 5H, Ar). FT-IR v $v_{\max } 2922$, 2854, 1659, $1515,1462,1260,1215,1026,808,761 \mathrm{~cm}^{-1}$.

1-(2,3-Dimethylphenyl)-6,6-dimethyl-2-phenyl-6,7-dihydro-1 $H$-indol$4(5 H)$-one $(5 e)$ :

White solid, yield $89 \%$, m.p. $147^{\circ} \mathrm{C}$ (dec.). ${ }^{1} \mathrm{H}-\mathrm{NMR}\left(300 \mathrm{MHz}, \mathrm{CDCl}_{3}\right) \delta$ (ppm): 1.09 (s, 3H, Me), 1.12 (s, 3H, Me), 1.76 (s, 3H, Me), 2.19 (d, $J=17.1$ $\mathrm{Hz}, 1 \mathrm{H}), 2.28(\mathrm{~s}, 3 \mathrm{H}, \mathrm{Me}), 2.33(\mathrm{~d}, J=16.2 \mathrm{~Hz}, 1 \mathrm{H}), 2.46(\mathrm{~d}, J=16.2 \mathrm{~Hz}, 1 \mathrm{H})$ $2.47(\mathrm{~d}, J=17.1 \mathrm{~Hz}, 1 \mathrm{H}), 6.84(\mathrm{~s}, 1 \mathrm{H}, \mathrm{Ar}), 7.27-7.07$ (m, 8H, Ar). ${ }^{13} \mathrm{C}-\mathrm{NMR}$ $\left(75.5 \mathrm{MHz}, \mathrm{CDCl}_{3}\right) \delta(\mathrm{ppm}): 13.96,20.33,27.99,29.22,35.58,36.59,52.20$, $104.87,119.66,126.21,126.84,127.46,127.69,128.15,130.52,132.12$ $134.76,136.65,136.84,138.63,145.06,193.93$. FT-IR v 2924, 2856, 1658 , $1468,1283,1219,1084,771,701 \mathrm{~cm}^{-1}$. Anal. Calc. for $\mathrm{C}_{24} \mathrm{H}_{25} \mathrm{NO}: \mathrm{C}, 83.93 ; \mathrm{H}$, 7.34; N, 4.08. Found: C, 83.88; H, 7.41; N, 7.28\%.

1-(3-Chloro-2-methylphenyl)-6,6-dimethyl-2-phenyl-6,7-dihydro-1Hindol-4(5H)-one $(5 f)$ :

Light pink solid, yield 77\%, m.p. $164{ }^{\circ} \mathrm{C} .{ }^{1} \mathrm{H}-\mathrm{NMR}\left(300 \mathrm{MHz}, \mathrm{CDCl}_{3}\right) \delta$ (ppm): 0.86 (s, 3H, Me), 1.05 (s, 3H, Me), 1.87 (s, 2H, $\left.\mathrm{CH}_{2}\right), 2.21$ (s, 3H, Me) $2.30\left(\mathrm{~s}, 2 \mathrm{H}, \mathrm{CH}_{2}\right), 6.97(\mathrm{t}, J=8.1 \mathrm{~Hz}, 1 \mathrm{H}, \mathrm{Ar}), 7.00-7.15(\mathrm{~m}, 6 \mathrm{H}, \mathrm{Ar}), 7.17(\mathrm{~d}$ $J=8.1 \mathrm{~Hz}, 1 \mathrm{H}, \mathrm{Ar}), 7.25(\mathrm{~d}, J=8.7 \mathrm{~Hz}, 1 \mathrm{H}, \mathrm{Ar}) \cdot{ }^{13} \mathrm{C}-\mathrm{NMR}\left(75.5 \mathrm{MHz}, \mathrm{CDCl}_{3}\right)$ $\delta$ (ppm): $15.34,27.46,28.76,29.70,31.71,46.37,110.00,112.70,125.91$, $127.27,127.68,128.14,128.86,128.97,129.77,131.55,134.85,135.12$, 135.43, 139.02, 186.23. FT-IR $v_{\text {max }} 3064,2956,2873,1618,1467,1398,1297$, $1151,1110,1016,767,700 \mathrm{~cm}^{-1}$. Anal. Calc. for $\mathrm{C}_{23} \mathrm{H}_{22}$ ClNO: C, 75.92; $\mathrm{H}$, $6.09 ; \mathrm{N}, 3.85$. Found: C, 76.12; H, 5.91; N, 3.73\%.

6,6-Dimethyl-1-(naphthalene-1-yl)-2-phenyl-6,7-dihydro- $1 H$-indol4(5H)-one (5g):

White solid, yield $73 \%$, m.p. $189^{\circ} \mathrm{C} .{ }^{1} \mathrm{H}-\mathrm{NMR}\left(300 \mathrm{MHz}, \mathrm{CDCl}_{3}\right) \delta(\mathrm{ppm})$ : 1.05 (s, 3H, Me), 1.06 (s, 3H, Me), 2.17 (d, $\left.J=16.5 \mathrm{~Hz}, 1 \mathrm{H}, \mathrm{CH}_{2}\right), 2.37$ (d, $J$ $\left.=16.5 \mathrm{~Hz}, 1 \mathrm{H}, \mathrm{CH}_{2}\right), 2.45\left(\mathrm{~s}, 2 \mathrm{H}, \mathrm{CH}_{2}\right), 6.93(\mathrm{~s}, 1 \mathrm{H}, \mathrm{Ar}), 7.04$ (br s, $\left.5 \mathrm{H}, \mathrm{Ar}\right)$, $7.38(\mathrm{t}, J=6.6 \mathrm{~Hz}, 2 \mathrm{H}, \mathrm{Ar}), 7.51(\mathrm{t}, J=7.2 \mathrm{~Hz}, 2 \mathrm{H}, \mathrm{Ar}), 7.56(\mathrm{t}, J=6.9 \mathrm{~Hz}$, $1 \mathrm{H}, \mathrm{Ar}), 7.95(\mathrm{~d}, J=7.8 \mathrm{~Hz}, 2 \mathrm{H}, \mathrm{Ar}){ }^{13} \mathrm{C}-\mathrm{NMR}(75.5 \mathrm{MHz}, \mathrm{CDCl}) \delta(\mathrm{ppm}):$ $28.06,28.89,35.54,36.38,52.21,105.21,119.88,122.63,125.28,126.55$, $126.92,127.54,127.78,128.07,128.42,128.75,129.41,130.94,131.89$, 134.09, 134.32, 137.59, 146.16, 194.07. FT-IR $v_{\text {max }} 3056,2955$, 2871, 1660, $1600,1554,1464,1412,1218,1148,758,695 \mathrm{~cm}^{-1}$. Anal. Calc. for $\mathrm{C}_{26} \mathrm{H}_{23} \mathrm{NO}$ :
C, 85.45; H, 6.34; N, 3.83. Found: C, 85.33; H, 6.45; N, 3.72\%.

\section{CONCLUSIONS}

In conclusion, we have successfully developed a facile, efficient procedure for the synthesis of 1-aryl-6,6-dimethyl-2-phenyl-6,7-dihydro- $1 \mathrm{H}$-indol$4(5 H)$-ones by two steps, including a three-component reaction in waterethanol under reflux. The advantageous features of this procedure are, high yields, operational simplicity, availability of starting materials and the use of water-ethanol as an environmentally friendly solvent.

\section{ACKNOWLEDGMENTS}

The authors gratefully acknowledge the financial assistance from Urmia University.

\section{REFERENCES}

1. N.K. Kaushik, N. Kaushik, P. Attri, N. Kumar, C.H. Kim, A.K. Verma, E.H. Choi, Molecules. 18, 6620 (2013).

2. R. Dhani, A. Avinash, S.K. Salenaagina, M.V.S. Teja, P. Masthanaiah, P.R. Rathnam, V.C. Silpa, J. Chem. Pharm. Res. 519 (2011).

3. H.B. Broughton, H.J. Bryant, M.S. Chambers, N.R. Curtis, WO 9962, 899, 1999; Chem. Abstr. 1999, 132: 12259y.

4. D.B. Fick, M.M. Foreman, A.J. Glasky, D.R. Helton, WO; 0311, 396, 2003; Chem. Abstr. 2003, 138: 163572v.

5. S. Suzen, E. Buyukbingol, Il Farmaco. 55, 246 (2000).

6. E. Buyukbingol, S. Suzen, G. Klopman, IlFarmaco. 49, 443 (1994).

7. C.C. Chiang, Y.H. Lin, S.F. Lin, C.L. Lai, C. Liu, W.Y. Wei, S.C. Yang, R.W. Wang, L.W. Teng, S.H. Chuang, J.M. Chang, T.T. Yuan, Y.S. Lee, P. Chen, W.K. Chi, J. Y. Yang, H.J. Huang, C.B. Liao, J.J. Huang, J. Med. Chem. 53, 5929 (2010).

8. P.M. Lieberman, A. Wolfler, P. Felsner, D. Hofer, K. Schauenstien, Arch. Allergy Immunol. 112, 203 (1997).

9. S. Suzen, E. Buyukbingol, IlFarmaco. 53, 525 (1998).

10. C.W. Zapf, J.D. Bloom, J.L. McBean, R.G. Dushin, T. Nittoli, C. Ingalls, A.G. Sutherland, J.P. Sonye, C.N. Eid, J. Golas, H. Liu, F. Boschelli, Y. Hu, E. Vogan, J.I. Levin, Bioorg. Med. Chem. Lett. 21, 2278 (2011).

11. K. Spyridonidou, M. Fousteris, M. Antonia, A. Chatzianastasiou, A. Papapetropoulos, S. Nikolaropoulos, Bioorg. Med. Chem. Lett. 19, 4810 (2009).

12. (a) M. Bandini, A. Melloni, A. Umani-Ronchi, Angew. Chem., Int. Ed. 43 , 550 (2004). (b) J.F. Austin, D.W.C. MacMillan, J. Am. Chem. Soc. 124, 1172 (2002). (c) K.B. Jensen, J. Thorhange, R.G. Hazel, K.A. Jorgensen, Angew. Chem., Int. Ed. 40, 160 (2001).

13. (a) N. Srivastava, B.K. Banik, J. Org. Chem. 68, 2109 (2003). (b) G. Bartoli, M. Bartolacci, M. Bosco, G. Foglia, A. Giuliani, E. Marcantoni, L. Sambri, E. Torregiani, J. Org. Chem. 68, 4594 (2003).

14. (a) N. Yoshiaki, Y. Masato, I. Youichi, H. Masnobu, U. Sakae, J. Am. Chem. Soc. 124, 11846 (2002). (b) E. Wenkert, E.C. Angell, V.F. Ferreira, E.L. Michelotti, S.R. Piettre, I. Chen, S. Safe, L. Bjeldanes, Biochem. Pharmacol. 51, 1069 (1996).

15. P. Ashok Gajapathi Raju, R. Mallikarjunarao, K. Venu Gopal, J. Sreeramulu, D. Maheswara Reddy, K.P. Krishnamurthi, S. Rajasekhar Reddy, J. Chem. Pharm. Res. 5, 21 (2013).

16. P.T. Anastas, J.C. Warner, Green Chemistry: Theory and Practice. Oxford University Press, New York, 1998.

17. D. Warren, Green Chemistry. A Teaching Resource. Royal Society of Chemistry, Cambridge, 2001.

18. J. Clark, D. Macquarrie, Handbook of Green Chemistry and Technology. Blackwell Publishing, Abingdon, Oxfordshire, 2002.

19. J. Sapi, J.Y. Laronze, Arkivoc. v, 208 (2004).

20. M. E. Huwaida Hassaneen, Arkivoc. i, 154 (2007).

21. For a monograph on MCRs, see: Multicomponent Reactions, ed. J. Zhu, H. Bienayme', Wiley-VCH, 2005.

22. Symposium in print on MCRs, see: Tetrahedron Symposia-in-Print, ed. I. Marek, 2005, vol. 67, p. 11299.

23. H. Batchu, S. Batra. Eur. J. Org. Chem., 2935 (2012).

24. S. Kaladevi, J. Sridhar, B. Abhilashamole, S. Muthusubramanian, N. Bhuvanesh, RSC Adv. 4, 34382 (2014).

25. J. Khalafy, N. Etivand, S. Dilmaghani, M. Ezzati, A. Poursattar Marjani, Tetrahedron Lett. 55, 3781 (2014). b) J. Khalafy, A. Poursattar Marjani, F. Salami, Tetrahedron Lett. 55, 6671 (2014). c) J. Khalafy, M. Ezzati, 
M. Rimaz, A. Poursattar Marjani, H. Yaghoobnejad Asl, J. Iran. Chem. Soc. 11, 1067 (2014). d) J. Khalafy, M. Mohammadlou, M. Mahmoody, F. Salami, A. Poursattar Marjani, Tetrahedron Lett. 56, 1528 (2015). e) A. Poursattar Marjani, J. Khalafy, F. Salami, M. Mohammadlou, Synthesis. 47, 1656 (2015). f) A. Poursattar Marjani, J. Khalafy, F. Salami, M. Ezzati, Arkivoc. v, 277 (2015). g) A. Poursattar Marjani, J. Khalafy, S. Mahmoodi. Arkivoc. iii, 262 (2016). h) A. Poursattar Marjani, J. Khalafy, A. Rostampoor. J. Heterocycl. Chem., in press (2016). http://onlinelibrary. wiley.com/doi/10.1002/jhet.2637/full.
26. K. Nagarajan, J. David, R.K. Shah, J. Med. Chem. 19, 508 (1976).

27. R. Mallikarjuna Rao, G. Nagaraja Reddy, J. Sreeramulu, Der Pharma Chemica. 3, 301 (2011).

28. P.N. Maini, M.P. Sammes, A.R. Katritzky, J. Chem. Soc., Perkin Trans. 1. 161 (1988)

29. S.R. Ramadas, S. Padmanabhan, J. Prakt. Chem. 320, 863 (1978).

30. P.B. Terentiev, Y.G. Boundel, A.N. Kost, B.I. Maksimov, J. Heterocyclic. Chem. 19, 645 (1982). 